\title{
A Nonclassical Radau Collocation Method for Nonlinear Initial-Value Problems with Applications to Lane-Emden Type Equations
}

\author{
Mohammad Maleki, ${ }^{1}$ M. Tavassoli Kajani, ${ }^{2}$ I. Hashim, $^{1}$ \\ A. Kilicman, ${ }^{3}$ and K. A. M. Atan ${ }^{4}$ \\ ${ }^{1}$ School of Mathematical Sciences, Universiti Kebangsaan Malaysia, 43600 Bangi, Selangor, Malaysia \\ ${ }^{2}$ Department of Mathematics, Islamic Azad University, Khorasgan Branch, Isfahan 71595, Iran \\ ${ }^{3}$ Department of Mathematics, Universiti Putra Malaysia, 43400 Serdang, Selangor, Malaysia \\ ${ }^{4}$ Institute for Mathematical Research (INSPEM), Universiti Putra Malaysia, 43400 Serdang, \\ Selangor, Malaysia \\ Correspondence should be addressed to A. Kilicman, akilicman@putra.upm.edu.my
}

Received 21 March 2012; Revised 16 September 2012; Accepted 16 September 2012

Academic Editor: Igor Andrianov

Copyright (C) 2012 Mohammad Maleki et al. This is an open access article distributed under the Creative Commons Attribution License, which permits unrestricted use, distribution, and reproduction in any medium, provided the original work is properly cited.

\begin{abstract}
We propose a numerical method for solving nonlinear initial-value problems of Lane-Emden type. The method is based upon nonclassical Gauss-Radau collocation points, and weighted interpolation. Nonclassical orthogonal polynomials, nonclassical Radau points and weighted interpolation are introduced on arbitrary intervals. Then they are utilized to reduce the computation of nonlinear initial-value problems to a system of nonlinear algebraic equations. We also present the comparison of this work with some well-known results and show that the present solution is very accurate.
\end{abstract}

\section{Introduction}

Many problems in the literature of mathematical physics can be formulated as equations of the Lane-Emden type defined in the form

$$
y^{\prime \prime}+\frac{2}{x} y^{\prime}+g(y(x))=0, \quad 0<x<\infty
$$

subject to

$$
y(0)=A, \quad y^{\prime}(0)=0,
$$


where prime denotes differentiation with respect to $x$. The solution of the Lane-Emden equation, as well as those of a variety of nonlinear problems in quantum mechanics and astrophysics such as the scattering length calculations in the variable phase approach, is numerically challenging because of the singular point at the origin. Equations (1.1) and (1.2) with specializing $g(y(x))$ and $A$ occur in several models of mathematical physics and astrophysics such as the theory of stellar structure, the thermal behavior of a spherical cloud of gas, and theories of thermionic currents. It has been studied widely in the literature; see, for example, [1-12]. This equation was first studied by the astrophysicists Jonathan Homer Lane and Robert Emden, which considered the thermal behavior of a spherical cloud of gas acting under the mutual attraction of its molecules and subject to the classical laws of thermodynamics [13]. For $g(y)=y^{\alpha}$, and $A=1$ in (1.1) and (1.2), we obtain the standard Lane-Emden equation of index $\alpha$ which has been the object of much study [1-3]. It was physically shown that interesting values of $\alpha$ lie in the interval $[0,5]$, and this equation has analytical solutions for $\alpha=0,1$ and 5 . Various alternative techniques have been developed for the solution of the Lane-Emden equation in the literature. Among others, this equation has been solved by means of perturbation methods and a $(1-1)$ Padé approximation (Bender et al. [2]), Adomian's decomposition method [3], the quasilinearization method of [4], the homotopy analysis method $[5,6]$, a variational approach which uses a semi-inverse method to obtain a variational principle [7], a power series solution [8], a linearization technique $[9,10]$, the variational iteration method $[11,12]$, hybrid functions collocation method [14], Lagrangian method [15], Hermite functions collocation method [16], Sinc-Collocation method [17], rational Legendre pseudospectral approach [18], a modified Legendre-spectral method [19], and a numerical technique based on converting the Lane-Emden equations into integral equations [20]. the form

In the present paper, we first consider the nonlinear ordinary differential equations of

$$
f\left(x, y(x), y^{\prime}(x), y^{\prime \prime}(x)\right)=0, \quad 0 \leqslant x<\infty
$$

with initial conditions

$$
y(0)=c_{1}, \quad y^{\prime}(0)=c_{2}
$$

We assume that (1.3) and (1.4) have a unique solution $y(x)$ to be determined. We then solve a variety of Lane-Emden equations which fall into this category. Here, we introduce a new direct computational method for solving (1.3) and (1.4). This method consists of reducing the solution of (1.3) and (1.4) to a set of algebraic equations by first interpolating $y(x)$ using weighted Lagrange interpolation based on Freud-type weights and sets of nonclassical Gauss-Radau (NGR) nodes. These nodes, which arise from nonclassical orthogonal polynomials based on Freud-type weights over interval $[a, T]$, are presented. Equation (1.3) is then collocated at these NGR collocation points to evaluate the unknown coefficients, which are the values of the function $y(x)$ at these collocation points.

This paper is organized as follows. In Section 2, we describe the generation of NGR collocation points, function approximation, and selection of weights. In Section 3, we explain our method, and in Section 4, the present method is applied to a nonlinear Lane-Emden equation as well as the standard Lane-Emden equation of index $\alpha$. The numerical solutions 
are compared in Section 5 with available exact or approximate solutions in order to assess the accuracy of the proposed method.

\section{Nonclassical Radau Collocation Method}

\subsection{NGR Points}

In classical pseudospectral methods [14, 22-24], the classical Gauss-Lobatto and GaussRadau collocation points are based on Chebyshev or Legendre polynomials and lie on the closed interval $[-1,1]$ and half-open interval $[-1,1)$, respectively. In the present work, we consider the generation of the NGR collocation points based on nonclassical orthogonal polynomials with respect to exponential weights in the intervals $[a, T]$ where $a$ and $T$ are any real numbers.

Let $N+1$ be the number of collocation points and let $P_{N}(t)$ be the $N$ th-degree nonclassical orthogonal polynomial with respect to weight $w(t)$ which can be obtained from the following three-term recurrence relation [25]:

$$
\begin{aligned}
& P_{k+1}(x)=\left(x-\alpha_{k}\right) P_{k}(x)-\beta_{k} P_{k-1}(x), \quad k=0,1,2, \ldots, \\
& P_{-1}(x)=0, \quad P_{0}(x)=1 .
\end{aligned}
$$

The recurrence coefficients in (2.1) are given in [26] by

$$
\begin{gathered}
\alpha_{k}=\frac{\int_{a}^{T} t w(x) P_{k}^{2}(x) d x}{\int_{a}^{T} w(x) P_{k}^{2}(x) d x}, \quad k=0,1,2, \ldots, \\
\beta_{0}=\int_{a}^{T} w(x) P_{0}^{2}(x) d x, \quad \beta_{k}=\frac{\int_{a}^{T} w(x) P_{k}^{2}(x) d x}{\int_{a}^{T} w(x) P_{k-1}^{2}(x) d x} .
\end{gathered}
$$

The NGR collocation points $x_{j}$ for $j=0,1, \ldots, N$ are obtained by the method outlined by Golub [27]. The tridiagonal Jacobi-Radau matrix of order $N+1$ is defined by

$$
J_{N+1}^{R}=\left(\begin{array}{ccccc}
\alpha_{0} & \sqrt{\beta_{1}} & & & \\
\sqrt{\beta_{1}} & \alpha_{1} & \sqrt{\beta_{2}} & & \\
& \sqrt{\beta_{2}} & \alpha_{2} & \sqrt{\beta_{3}} & \\
& \ddots & \ddots & \ddots & \\
& & \sqrt{\beta_{N-1}} & \alpha_{N-1} & \sqrt{\beta_{N}} \\
& & & \sqrt{\beta_{N}} & \alpha_{N}^{R}
\end{array}\right),
$$


where $\alpha_{N}^{R}$ is obtained from

$$
\alpha_{N}^{R}=a-\beta_{N} \frac{P_{N-1}(a)}{P_{N}(a)}
$$

Theorem 2.1 (see Golub [27]). The Gauss-Radau nodes $a=x_{0}<x_{1}<\cdots<x_{N}<T$ are the eigenvalues of $J_{N+1}^{R}$, and the Gauss-Radau weights $w_{j}$ are given by

$$
w_{j}=\beta_{0}\left(v_{1 j}\right)^{2}, \quad j=0,1, \ldots, N,
$$

where $v_{j}$ is the normalized eigenvector of $J_{N+1}^{R}$ corresponding to the eigenvalue $x_{j}\left(i . e ., v_{j}^{T} v_{j}=1\right)$ and $v_{1 j}$ its first component.

\subsection{Function Approximation and Differentiation Matrices}

Consider the NGR collocation points $\left(x_{0}, x_{1}, \ldots, x_{N}\right)$ defined in Section 2.1 on the interval $[a, T)$ and additional noncollocated point $x_{N+1}=T$. The function $y \in L^{2}[a, T]$ is approximated by weighted Lagrange interpolation as $[26,28]$

$$
y(x) \approx y_{N}(x)=\sum_{j=0}^{N+1} \frac{W(x)}{W\left(x_{j}\right)} L_{j}(x) y_{j}, \quad x \in[a, T],
$$

where $W$ is a positive and bounded weight function, and $y_{j}=y\left(x_{j}\right)$,

$$
L_{j}(x)=\prod_{\substack{k=0 \\ k \neq j}}^{N+1} \frac{x-x_{k}}{x_{j}-x_{k}}, \quad j=0,1, \ldots, N+1,
$$

is a basis of $(N+1)$ th-degree Lagrange polynomials. Notice that the basis includes the function $L_{N+1}$ corresponding to the terminal value $x_{N+1}=T$. Differentiating twice the series of (2.6) and evaluating the NGR collocation points $x_{i}, 0 \leqslant i \leqslant N$ give

$$
\begin{aligned}
& y^{\prime}\left(x_{i}\right) \approx \sum_{j=0}^{N+1} y_{j} \frac{d}{d x}\left[\frac{W(x)}{W\left(x_{j}\right)} L_{j}(x)\right]_{x=x_{i}}=D_{i}^{(1)} \cdot Y, \\
& y^{\prime \prime}\left(x_{i}\right) \approx \sum_{j=0}^{N+1} y_{j} \frac{d^{2}}{d x^{2}}\left[\frac{W(x)}{W\left(x_{j}\right)} L_{j}(x)\right]_{x=x_{i}}=D_{i}^{(2)} \cdot Y,
\end{aligned}
$$

where

$$
Y=\left[y_{0}, y_{1}, \ldots, y_{N+1}\right]^{T},
$$


$D_{i}^{(1)}$ and $D_{i}^{(2)}$ are the $i$ th row of $\mathbf{D}^{(1)}$ and $\mathbf{D}^{(2)}$,

$$
\begin{aligned}
& D_{i j}^{(1)}=\frac{d}{d x}\left[\frac{W(x)}{W\left(x_{j}\right)} L_{j}(x)\right]_{x=x_{i}}, \\
& D_{i j}^{(2)}=\frac{d^{2}}{d x^{2}}\left[\frac{W(x)}{W\left(x_{j}\right)} L_{j}(x)\right]_{x=x_{i}} .
\end{aligned}
$$

The rectangular $(N+1) \times(N+2)$ matrices $\mathbf{D}^{(1)}$ and $\mathbf{D}^{(2)}$ formed by the coefficients $D_{i j}^{(1)}$ and $D_{i j}^{(2)}, i=0,1, \ldots, N ; j=0,1, \ldots, N+1$ are the first and second order Gauss-Radau differentiation matrices, respectively. These matrices transform the approximation of $y(x)$ at $x_{0}, \ldots, x_{N+1}$ to the first and second derivatives of $y$ at the collocation points $x_{0}, \ldots, x_{N}$.

\subsection{Weight Selection}

When studying the uniform convergence behavior of the weighted Lagrange interpolation as $N \rightarrow \infty$, a crucial role is played by the Lebesgue function

$$
\lambda_{N, W}(x)=W(x) \sum_{j=0}^{N+1} \frac{\left|L_{j}(x)\right|}{W\left(x_{j}\right)},
$$

and the Lebesgue constant

$$
\Lambda_{N, W}=\left\|\Lambda_{N, W}(x)\right\|_{\infty}
$$

(see, e.g., [29-32] and references therein).

In general, the orthogonal weight function $w$ and weight of interpolation $W$ are chosen independently [26, 28, 33-35]. Nevertheless, if we expect a reasonable upper estimate for the Lebesgue constant, then we have to assume some connections between these two weights. The most natural assumption, as suggested in [29], is to generate the interpolation nodes (collocation points) $x_{j}$ with respect to the weight $w=W^{2}$. In addition, to obtain uniform convergence in weighted interpolation some conditions on $W$ should be considered. The Bernstein's approximation problem deals with the uniform convergence behavior of weighted interpolation. The problem is as follows

Let $W>0$ be measurable. When it is true that for every continuous $y: \mathbb{R} \rightarrow \mathbb{R}$ with

$$
\lim _{|x| \rightarrow \infty} W(x) y(x)=0
$$

there exist a sequence of polynomials $\left\{F_{N}\right\}_{N=1}^{\infty}$ with

$$
E_{N}(y)_{W}=\lim _{N \rightarrow \infty}\left\|W\left(y-F_{N}\right)\right\|=0 ?
$$

The condition $\lim _{|x| \rightarrow \infty} W(x) y(x)=0$ is essential to counteract the growth of any polynomial at infinity. 
As stated above, the Lebesgue constant plays an important role in answering the Bernstein's problem. Now it is known that for any set of interpolation nodes and any weight $W, \Lambda_{N, W}$ is unbounded with respect to $N$. A consequence of this is that there exists $y$ such that weighted interpolation does not converge uniformly to $y$. However, if $y$ is not too badly behaved (e.g., as measured by the modulus of continuity) and the $\Lambda_{N, W}$ are not too large, then uniform convergence is achieved (as positive answers to the Bernstein's problem).

The Freud-type weights, as positive answers to the Bernstein's problem, (see, e.g., [36]) are defined as $W(t)=\exp (-Q(t))$ with the following conditions: $Q: \mathbb{R} \rightarrow \mathbb{R}$ is even, continuous in $\mathbb{R}, Q^{\prime \prime}$ is continuous in $(0, \infty), Q^{\prime}>0$ on $(0, \infty)$ and for some $1<a \leq b$

$$
a \leq \frac{\left(x Q^{\prime}(x)\right)^{\prime}}{Q^{\prime}(x)} \leq b, \quad x \in(0, \infty)
$$

If $W$ is a Freud weight we write $W \in \mathcal{F}$, and if, moreover, $Q$ is differentiable in $\mathbb{R}$, we write $w \in \mathcal{F} 1$. By definition, if $W \in \mathcal{F}$, then $W^{2} \in \mathcal{F}$, too. Canonical example is as

$$
W_{\beta}(x)=\exp \left(-|x|^{\beta}\right), \quad \beta \geq 1, x \in \mathbb{R}
$$

Clearly $W_{\beta} \in \mathcal{F} 1$ with $a=b=\beta$. In this work, we consider the Freud weight with $\beta=1$.

Vértesi in [32] has shown that given a Freud-type weight satisfying the previous conditions and any set of interpolation nodes

$$
\log N=O\left(\Lambda_{N, W}\right), \quad N \rightarrow \infty
$$

\section{Solution of Nonlinear Initial-Value Problems}

Our discrete approximation to the nonlinear initial-value problem in (1.3)-(1.4) is obtained by evaluating (2.6) at $N$ collocation points $x_{1}, \ldots, x_{N}$ and replacing $y^{\prime}\left(x_{i}\right)$ and $y^{\prime \prime}\left(x_{i}\right)$ by their discrete approximations in (2.8), and evaluating the boundary conditions in (1.4) at collocation point $x_{0}=0$. Hence, the discrete approximation to the nonlinear initial-value problem is

$$
\begin{aligned}
& f\left(x_{i}, y_{i}, D_{i}^{(1)} \cdot Y, D_{i}^{(2)} \cdot Y\right)=0, \quad i=1,2, \ldots, N, \\
& y\left(x_{0}\right)=y_{0}=c_{1}, \quad D_{0}^{(1)} \cdot Y=c_{2} .
\end{aligned}
$$

Using (3.1) and (3.2), we obtain a system of $N+2$ nonlinear algebraic equations which can be solved using the Newton's iterative method.

It is well known that the initial guess for Newton's iterative method is very important especially for complicated problems. To choose the initial guess for our problem, in the first stage we set $N=5$ and apply the Newton's iterative method for solving $N+2$ nonlinear equations by choosing $c_{1}$ in (1.4) as our initial guess. We then increase $N$ by 5 and use the approximate solution in stage one as our initial guess in this stage. We continue this approach until the results are similar up to a required number of decimal places for two consecutive stages. 
It is worth mentioning that, in the case that the initial-value problem has a singularity at $x=0$ (e.g., the Lane-Emden type equations), this method avoids the singularity, because we compute (3.1) at the collocation points that are straightly more than zero.

\section{Illustrative Examples}

We applied the method presented in this paper and solved two problems. The first example is a nonlinear Lane-Emden equation considered in [10] and the second example is the standard Lane-Emden equation of index $\alpha$. As stated in Section 2.3, we consider the Freud-type weight $W(x)=\exp (-|x|)$ as interpolation weight function and generate the nonclassical orthogonal polynomials and the NGR collocation points in the intervals $[0, T]$ with respect to the weight $w=W^{2}$.

Example 4.1. This example corresponds to the following singular nonlinear Lane-Emden equation [10]:

$$
\begin{aligned}
& y^{\prime \prime}+\frac{2}{x} y^{\prime}+4\left(2 e^{y}+e^{y / 2}\right)=0, \quad 0<x<\infty \\
& y(0)=0, \quad y^{\prime}(0)=0
\end{aligned}
$$

which has the following exact solution:

$$
y_{e}(x)=-2 \ln \left(1+x^{2}\right)
$$

Define

$$
\begin{aligned}
& E_{N, T}=\max \left\{\left|y_{e}(x)-y_{N}(x)\right|: 0 \leqslant x \leqslant T\right\} \\
& \widetilde{E}_{N, T}=\max \left\{\left|y_{e}^{\prime}(x)-y_{N}^{\prime}(x)\right|: 0 \leqslant x \leqslant T\right\}
\end{aligned}
$$

where $y_{N}(x)$ and $y_{e}(x)$ denote the approximate solution obtained by the present method and the exact solution, respectively. In Table 1, the maximum absolute errors between approximate and exact solutions are denoted by $E_{N, T}$, and maximum absolute error between the derivative of approximate and exact solutions is denoted by $\widetilde{E}_{N, T}$, for different values of $N$ and $T$ are given, which show the efficiency of the present method in large interval calculation. Further, in Table 2 a comparison is made between the values of $y(x)$ obtained using the present method for $T=10$ and $N=30$ together with the values given in [16] using Hermite functions collocation method and the exact solutions.

Example 4.2. Consider the standard Lane-Emden equation of index $\alpha$ given by

$$
\begin{aligned}
& y^{\prime \prime}+\frac{2}{x} y^{\prime}+y^{\alpha}=0, \quad 0<x<\infty, \\
& y(0)=1, \quad y^{\prime}(0)=0,
\end{aligned}
$$


Table 1: The maximum absolute errors $E_{N, T}$ and $\widetilde{E}_{N, T}$ for Example 4.1.

\begin{tabular}{llcc}
\hline$T$ & $N$ & $E_{N, T}$ & $\widetilde{E}_{N, T}$ \\
\hline \multirow{2}{*}{20} & 20 & $1.80 \times 10^{-4}$ & $4.40 \times 10^{-4}$ \\
& 25 & $1.15 \times 10^{-5}$ & $1.50 \times 10^{-4}$ \\
& 30 & $1.25 \times 10^{-6}$ & $9.20 \times 10^{-6}$ \\
\hline \multirow{3}{*}{10} & 20 & $8.50 \times 10^{-6}$ & $1.25 \times 10^{-4}$ \\
& 25 & $4.10 \times 10^{-7}$ & $1.24 \times 10^{-5}$ \\
& 30 & $3.65 \times 10^{-8}$ & $4.50 \times 10^{-7}$ \\
\hline \multirow{2}{*}{5} & 20 & $1.85 \times 10^{-7}$ & $4.30 \times 10^{-6}$ \\
& 25 & $5.40 \times 10^{-9}$ & $1.83 \times 10^{-7}$ \\
& 30 & $1.60 \times 10^{-10}$ & $7.50 \times 10^{-9}$ \\
\hline
\end{tabular}

Table 2: Comparison of $y(x)$ between present method, method in [16] and the exact values for Example 4.1.

\begin{tabular}{lccccc}
\hline$x$ & Method in [16] & Error & Present method & Error & Exact \\
\hline 0.00 & 0.0000000000 & $0.00 \times 10^{0}$ & 0.0000000000 & $0.00 \times 10^{0}$ & 0.0000000000 \\
0.01 & -0.0001970587 & $2.93 \times 10^{-6}$ & -0.0001999904 & $4.08 \times 10^{-10}$ & -0.0001999900 \\
0.10 & -0.0198967225 & $3.94 \times 10^{-6}$ & -0.0199006640 & $2.27 \times 10^{-9}$ & -0.0199006617 \\
0.50 & -0.4462840851 & $3.02 \times 10^{-6}$ & -0.4462870994 & $3.28 \times 10^{-9}$ & -0.4462871026 \\
1.00 & -1.3862934297 & $9.31 \times 10^{-7}$ & -1.3862943490 & $1.21 \times 10^{-8}$ & -1.3862943611 \\
2.00 & -3.2188763248 & $5.00 \times 10^{-7}$ & -3.2188758411 & $1.63 \times 10^{-8}$ & -3.2188758249 \\
3.00 & -4.6051709964 & $8.10 \times 10^{-7}$ & -4.6051702055 & $1.95 \times 10^{-8}$ & -4.6051701860 \\
4.00 & -5.6664274573 & $7.69 \times 10^{-7}$ & -5.6664266969 & $8.80 \times 10^{-9}$ & -5.6664266881 \\
5.00 & -6.5161937402 & $6.64 \times 10^{-7}$ & -6.5161930821 & $6.09 \times 10^{-9}$ & -6.5161930760 \\
6.00 & -7.2218363729 & $5.48 \times 10^{-7}$ & -7.2218358310 & $5.68 \times 10^{-9}$ & -7.2218358253 \\
7.00 & -7.8240461812 & $1.70 \times 10^{-7}$ & -7.8240460154 & $4.57 \times 10^{-9}$ & -7.8240460109 \\
8.00 & -8.3487734467 & $1.09 \times 10^{-7}$ & -8.3487745418 & $1.97 \times 10^{-9}$ & -8.3487745398 \\
9.00 & -8.8134506165 & $1.21 \times 10^{-5}$ & -8.8134384925 & $2.05 \times 10^{-9}$ & -8.8134384945 \\
10.00 & -9.2302027821 & $3.83 \times 10^{-5}$ & -9.2302410337 & $1.18 \times 10^{-11}$ & -9.2302410337 \\
\hline
\end{tabular}

This equation is linear for $\alpha=0$ and 1 , nonlinear otherwise, and exact solutions exist only for $\alpha=0,1$ and 5 and are given in Bender et al. [2], respectively, by

$$
y(x)=1-\frac{x^{2}}{6}, \quad y(x)=\frac{\sin (x)}{x}, \quad y(x)=\left(1+\frac{x^{2}}{3}\right)^{-1 / 2}
$$

Moreover, Bender et al. [2] determined the zeros of $y(x)$ asymptotically, here denoted by $\xi$, and found that

$$
\xi=\pi+0.885273956 \delta+0.24222 \delta^{2}
$$

for $\delta=-0.5,0,0.5,1.0$ and 1.5 which correspond to $\alpha=0,1,2,3$, and 4 , respectively.

We applied the method presented in this paper and solved this example and then evaluated the zeros of $y(x)$, which are also evaluated in [10] using linearization technique, in [15] by using Lagrangian method, and in [16] using Hermite functions collocation method. 


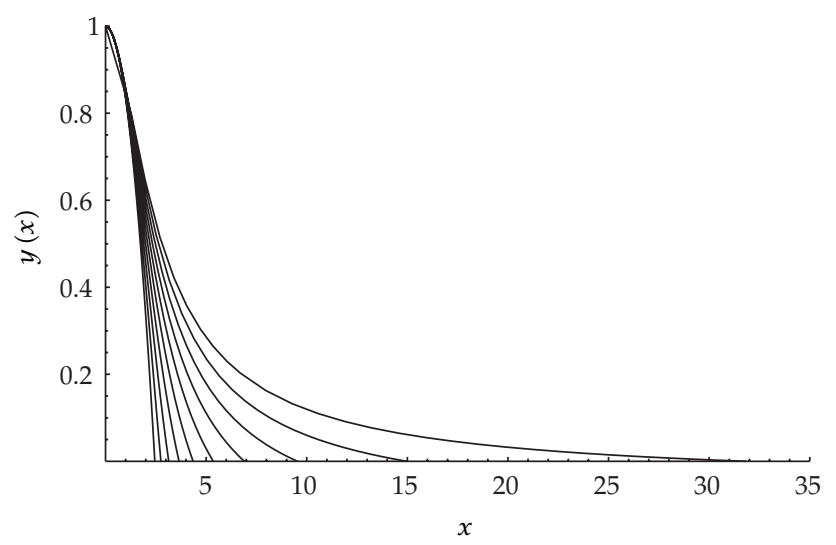

Figure 1: Lane-Emden graphs obtained by the present method for $\alpha=0,0.5,1, \ldots, 4.5$.

The selection of $T$ is crucial for the computing of zero, $\xi$, of $y(x)$. In order to obtain reasonable approximations of zeros, for each value of $\alpha$, in the first stage we select a sufficiently large $T$ and set $N=25$ then we solve the problem using the present method to obtain $\xi_{1}$ as the first approximation of the zero. Then according to the obtained $\xi_{1}$ we select a value for $T$. Finally, we resolve the problem for different values of $N$.

In Table 3, the resulting values of the zeros of $y(x)$ for $\alpha=0,0.5, \ldots, 4.5$ using the present method for different $N$, with the results obtained in $[10,15,16]$ together with the exact solutions of Horedt [21], are presented. Table 3 shows that the present method provides very accurate predictions of the zeros of $y(x)$ even in large intervals. In order to demonstrate the accuracy of the proposed method, in Tables 4 and 5 we have compared the numerical results of $y(x)$ using the present method for $\alpha=3, N=25$ and $\alpha=4, N=30$, respectively, with the methods in $[15-17,21]$. In addition, Table 6 shows the maximum absolute errors for $\alpha=5$, for which the exact solution exists, with $N=30$ and choosing different values of $T$. As can be seen from Tables 3-6, the present method provides very accurate results even for large values of $T$. The resulting graphs of the standard Lane-Emden equation for $\alpha=0,0.5,1,1.5$, $2,2.5,3,3.5,4$ and 4.5 and different values of $N$ given in Table 3 are shown in Figure 1.

\section{Comparison with Other Methods}

As stated in the Introduction section, the Lane-Emden equations have been widely solved using both numerical and analytical methods. In this section we aim to present the advantages of our numerical method over some other existing methods in the literature.

(i) Comparison with some analytical solutions: among others, the Lane-Emden equations have been solved with the variational iteration method [11, 12], homotopy-perturbation method [5, 6], and the Adomian decomposition method [3]. However, this type of solution methods is dependent on the initial guess so that the obtained series solution is changed with changing the initial guess, whereas the present method is not dependent on the initial guess. Furthermore, in the mentioned methods the interval of convergence of the obtained series solution is limited (usually $[0,1]$ ), whereas Section 4 shows that our method provides accurate 
Table 3: Comparison of numerical results for $\xi(y(\xi)=0)$ for Example 4.2.

\begin{tabular}{lcccccc}
\hline$\alpha$ & $N$ & Present method & Method in [10] & Method in [15] & Method in [16] & Exact [21] \\
\hline 0 & 20 & 2.4494897427832 & 2.44899 & - & - & 2.4494897427832 \\
0.5 & 27 & 2.752698051 & - & - & - & 2.75269805 \\
1 & 20 & 3.1415926535898 & 3.14048 & - & - & 3.1415926535898 \\
1.5 & 25 & 3.65375374 & - & - & 3.65375374 & 3.65375374 \\
2 & 15 & 4.35287460 & 4.35086 & 4.352875 & 4.35287460 & 4.35287460 \\
2.5 & 17 & 5.35527546 & - & - & 5.35527546 & 5.35527546 \\
3 & 25 & 6.89684862 & 6.89312 & 6.896846 & 6.89684862 & 6.89684862 \\
3.5 & 25 & 9.53580535 & - & - & - & 9.53580534 \\
4 & 30 & 14.9715464 & 14.96518 & 14.971546 & 14.9715463 & 14.9715463 \\
4.5 & 30 & 31.8364633 & - & - & - & 31.8364632 \\
\hline
\end{tabular}

Table 4: Comparison of $y(x)$ for present method, and other methods in the literature for $\alpha=3$.

\begin{tabular}{lcccccc}
\hline$x$ & Present method & Error & Error in [17] & Error in [15] & Error in [16] & Exact [21] \\
\hline 0.000 & 1.0000000000 & $0.00 \times 10^{0}$ & $0.00 \times 10^{0}$ & $0.00 \times 10^{0}$ & $0.00 \times 10^{0}$ & 1.0000000000 \\
0.100 & 0.9983358295 & $2.95 \times 10^{-8}$ & $1.82 \times 10^{-5}$ & $1.28 \times 10^{-5}$ & $1.40 \times 10^{-6}$ & 0.9983358000 \\
0.500 & 0.9598390699 & $3.00 \times 10^{-8}$ & $1.12 \times 10^{-4}$ & $1.81 \times 10^{-5}$ & $2.99 \times 10^{-6}$ & 0.9598391000 \\
1.000 & 0.8550575686 & $3.14 \times 10^{-8}$ & $1.07 \times 10^{-4}$ & $6.00 \times 10^{-7}$ & $1.99 \times 10^{-6}$ & 0.8550576000 \\
5.000 & 0.1108198351 & $3.51 \times 10^{-8}$ & $2.02 \times 10^{-4}$ & $2.00 \times 10^{-7}$ & $3.90 \times 10^{-7}$ & 0.1108198000 \\
6.000 & 0.0437379839 & $3.90 \times 10^{-9}$ & $5.00 \times 10^{-5}$ & $2.00 \times 10^{-5}$ & $1.14 \times 10^{-6}$ & 0.0437379800 \\
6.800 & 0.0041677894 & $4.00 \times 10^{-10}$ & $2.93 \times 10^{-4}$ & $2.79 \times 10^{-6}$ & $1.05 \times 10^{-5}$ & 0.0041677890 \\
6.896 & 0.0000360111 & $1.00 \times 10^{-10}$ & $2.10 \times 10^{-5}$ & $1.01 \times 10^{-6}$ & $8.88 \times 10^{-8}$ & 0.0000360112 \\
\hline
\end{tabular}

Table 5: Comparison of $y(x)$ for present method, and other methods in the literature for $\alpha=4$.

\begin{tabular}{lcccc}
\hline$x$ & Present method & Error & Error in [16] & Exact [21] \\
\hline 0.0 & 1.0000000000 & $0.00 \times 10^{0}$ & $0.00 \times 10^{0}$ & 1.0000000000 \\
0.1 & 0.9983366596 & $4.04 \times 10^{-8}$ & $2.51 \times 10^{-4}$ & 0.9983367000 \\
0.2 & 0.9933862134 & $1.34 \times 10^{-8}$ & $2.48 \times 10^{-4}$ & 0.9933862000 \\
0.5 & 0.9603109024 & $2.36 \times 10^{-9}$ & $2.05 \times 10^{-4}$ & 0.9603109000 \\
1.0 & 0.8608138124 & $1.24 \times 10^{-8}$ & $1.93 \times 10^{-4}$ & 0.8608138000 \\
5.0 & 0.2359227314 & $3.14 \times 10^{-8}$ & $8.59 \times 10^{-5}$ & 0.2359227000 \\
10.0 & 0.0596727418 & $1.80 \times 10^{-9}$ & $6.22 \times 10^{-5}$ & 0.0596727400 \\
14.0 & 0.0083305270 & $5.30 \times 10^{-12}$ & $2.47 \times 10^{-5}$ & 0.0083305270 \\
14.9 & 0.0005764180 & $9.09 \times 10^{-10}$ & $5.00 \times 10^{-7}$ & 0.0005764189 \\
\hline
\end{tabular}

Table 6: The maximum absolute errors $E_{N, T}$ and $\widetilde{E}_{N, T}$ for $\alpha=5$ and $N=30$.

\begin{tabular}{lcc}
\hline$T$ & $E_{N, T}$ & $\widetilde{E}_{N, T}$ \\
\hline 40 & $8.0 \times 10^{-6}$ & $1.0 \times 10^{-5}$ \\
30 & $5.5 \times 10^{-8}$ & $6.4 \times 10^{-7}$ \\
20 & $6.6 \times 10^{-9}$ & $1.1 \times 10^{-7}$ \\
10 & $1.4 \times 10^{-10}$ & $3.5 \times 10^{-9}$ \\
5 & $1.5 \times 10^{-13}$ & $3.2 \times 10^{-12}$ \\
\hline
\end{tabular}


approximate solutions in larger domains. For instance, consider Example 4.1 of Section 4 . The series solution for this example in $[3,6,12]$ is as follows:

$$
y(x)=-2\left(x^{2}-\frac{1}{2} x^{4}+\frac{1}{3} x^{6}-\frac{1}{4} x^{8}+\cdots\right),
$$

which converges to the exact solution only on the interval $[0,1]$, while we have solved this example with high accuracy in the interval $[0,20]$.

Note that in the mentioned analytical solutions, Padé approximant can be implemented for manipulating a polynomial approximation into a rational function to gain more information about the approximate solution. Nevertheless, comparison between Figure 1 of Wazwaz [3] obtained using Padé approximants [6/6] with Figure 1 and Table 3 of this paper shows that our method provides much more accurate predictions of the zeros of the standard Lane-Emden equation in Example 4.2.

(ii) Comparison with some spectral methods: several spectral methods have been established for solving the Lane-Emden equations [15-20]. Methods in [19, 20] can only be implemented in the interval $[0,1]$, while our method can be implemented in larger interval. Further, methods in [15-18] are based on orthogonal functions on the semi-infinite interval $[0, \infty]$, such as Laguerre functions, Hermite functions, and radial basis functions. All of these methods need certain quadratures on unbounded domains, which introduce errors and so weaken the merit of spectral approximations. Moreover, for an infinitely smooth function $f \in L^{2}(0,+\infty)$, the spectral convergence of the truncated series in these functions occurs only if $f$ decays exponentially fast at $+\infty$. According to these reasons, we see from Tables 2-5 that our method provides more accurate numerical results. Also, Table 3 shows that the spectral methods in [15-18] can solve the standard Lane-Emden equation up to the interval $[0,15]$, whereas we have solved this equation with high accuracy up to the interval $[0,32]$.

(iii) The present method has also the following advantages: first, for another type of equations the Freud-type weights described in Section 2.3 can be tuned to improve the accuracy of the discrete approximation. Second, this method provides very accurate results with moderate number of collocation points even in large intervals. Third, methods in [26, 28,33-35], by considering some arbitrary weight functions, have utilized nonclassical basis polynomials on the interval $[0,1]$. But, in the present work we have developed this idea over an arbitrary interval $[0, T]$ based on the Freud-type weights.

\section{Conclusion}

The Lane-Emden equation occurs in the theory of stellar structure and describes the temperature variation of a spherical gas cloud. The difficulty in this type of equations, due to the existence of singular point at $x=0$, is overcome here. In the standard Lane-Emden equation, the first zero of $y$ is an important point of the function, so we have computed $y$ up to this zero by utilizing the nonclassical Radau collocation method. A set of nonclassical orthogonal polynomials based on Freud-type weights is proposed to provide an effective but simple 
way to improve the convergence of the solution by a Radau collocation method. Numerical examples demonstrate the validity and high accuracy of the technique.

\section{Acknowledgments}

The present paper was prepared during the second author's visit to Institute for Mathematical Research (INSPEM), UPM. The second author thanks the staff of INSPEM for the kind hospitality.

\section{References}

[1] S. Chandrasekhar, An Introduction to the Study of Stellar Structure, Dover, New York, NY, USA, 1967.

[2] C. M. Bender, K. A. Milton, S. S. Pinsky, and L. M. Simmons, Jr., "A new perturbative approach to nonlinear problems," Journal of Mathematical Physics, vol. 30, no. 7, pp. 1447-1455, 1989.

[3] A.-M. Wazwaz, "A new method for solving singular initial value problems in the second-order ordinary differential equations," Applied Mathematics and Computation, vol. 128, no. 1, pp. 45-57, 2002.

[4] V. B. Mandelzweig and F. Tabakin, "Quasilinearization approach to nonlinear problems in physics with application to nonlinear ODEs," Computer Physics Communications, vol. 141, no. 2, pp. 268-281, 2001.

[5] S. Liao, "A new analytic algorithm of Lane-Emden type equations," Applied Mathematics and Computation, vol. 142, no. 1, pp. 1-16, 2003.

[6] M. S. H. Chowdhury and I. Hashim, "Solutions of Emden-Fowler equations by homotopy-perturbation method," Nonlinear Analysis. Real World Applications, vol. 10, no. 1, pp. 104-115, 2009.

[7] J.-H. He, "Variational approach to the Lane-Emden equation," Applied Mathematics and Computation, vol. 143, no. 2-3, pp. 539-541, 2003.

[8] M. I. Nouh, "Accelerated power series solution of polytropic and isothermal gas spheres," New Astronomy, vol. 9, pp. 467-473, 2004.

[9] J. I. Ramos, "Linearization methods in classical and quantum mechanics," Computer Physics Communications, vol. 153, no. 2, pp. 199-208, 2003.

[10] J. I. Ramos, "Linearization techniques for singular initial-value problems of ordinary differential equations," Applied Mathematics and Computation, vol. 161, no. 2, pp. 525-542, 2005.

[11] M. Dehghan and F. Shakeri, "Approximate solution of a differential equation arising in astrophysics using the variational iteration method," New Astronomy, vol. 13, pp. 53-59, 2008.

[12] A. Yıldırım and T. Öziş, "Solutions of singular IVPs of Lane-Emden type by the variational iteration method," Nonlinear Analysis. Theory, Methods \& Applications, vol. 70, no. 6, pp. 2480-2484, 2009.

[13] H. T. Davis, Introduction to Nonlinear Differential and Integral Equations, Dover, New York, ,NY, USA, 1962.

[14] H. R. Marzban, H. R. Tabrizidooz, and M. Razzaghi, "Hybrid functions for nonlinear initial-value problems with applications to Lane-Emden type equations," Physics Letters A, vol. 372, no. 37, pp. 5883-5886, 2008.

[15] K. Parand, A. R. Rezaei, and A. Taghavi, "Lagrangian method for solving Lane-Emden type equation arising in astrophysics on semi-infinite domains," Acta Astronautica, vol. 67, p. 673, 2010.

[16] K. Parand, M. Dehghan, A. R. Rezaei, and S. M. Ghaderi, "An approximation algorithm for the solution of the nonlinear Lane-Emden type equations arising in astrophysics using Hermite functions collocation method," Computer Physics Communications, vol. 181, no. 6, pp. 1096-1108, 2010.

[17] K. Parand and A. Pirkhedri, "Sinc-Collocation method for solving astrophysics equations," New Astronomy, vol. 15, pp. 533-537, 2010.

[18] K. Parand, M. Shahini, and M. Dehghan, "Rational Legendre pseudospectral approach for solving nonlinear differential equations of Lane-Emden type," Journal of Computational Physics, vol. 228, no. 23, pp. 8830-8840, 2009.

[19] H. Adibi and A. M. Rismani, "On using a modified Legendre-spectral method for solving singular IVPs of Lane-Emden type," Computers E Mathematics with Applications, vol. 60, no. 7, pp. 2126-2130, 2010.

[20] S. Karimi Vanani and A. Aminataei, "On the numerical solution of differential equations of LaneEmden type," Computers \& Mathematics with Applications, vol. 59, no. 8, pp. 2815-2820, 2010. 
[21] G. P. Horedt, Polytropes Applications in Astrophysics and Related Fields, Kluwer Academic Publishers, Dordrecht, The Netherlands, 2004.

[22] G. Elnagar, M. A. Kazemi, and M. Razzaghi, "The pseudospectral Legendre method for discretizing optimal control problems," IEEE Transactions on Automatic Control, vol. 40, no. 10, pp. 1793-1796, 1995.

[23] G. N. Elnagar and M. A. Kazemi, "Pseudospectral Chebyshev optimal control of constrained nonlinear dynamical systems," Computational Optimization and Applications, vol. 11, no. 2, pp. 195-217, 1998.

[24] D. Garg, M. Ptterson, W. W. Hager, A. V. Rao, and D. A. Benson, "A unified framework for the numerical solution of optimal control problems using pseudospectral methods," Automatica, vol. 46, p. 1843, 2010.

[25] B. Shizgal, "A Gaussian quadrature procedure for use in the solution of the Boltzmann equation and related problems," Journal of Computational Physics, vol. 41, no. 2, pp. 309-328, 1981.

[26] B. Shizgal and H. Chen, "The quadrature discretization method (QDM) in the solution of the Schrödinger equation with nonclassical basis functions," Journal of Chemical Physics, vol. 104, 4137 pages, 1996.

[27] G. H. Golub, "Some modified matrix eigenvalue problems," SIAM Review, vol. 15, pp. 318-334, 1973.

[28] M. Maleki and M. Mashali-Firouzi, "A numerical solution of problems in calculus of variation using direct method and nonclassical parameterization," Journal of Computational and Applied Mathematics, vol. 234, no. 5, pp. 1364-1373, 2010.

[29] J. Szabados, "Weighted Lagrange and Hermite-Fejér interpolation on the real line," Journal of Inequalities and Applications, vol. 1, no. 2, pp. 99-123, 1997.

[30] S. B. Damelin, "The asymptotic distribution of general interpolation arrays for exponential weights," Electronic Transactions on Numerical Analysis, vol. 13, pp. 12-21, 2002.

[31] D. S. Lubinsky, "A survey of weighted polynomial approximation with exponential weights," Surveys in Approximation Theory, vol. 3, pp. 1-105, 2007.

[32] P. Vértesi, "On the Lebesgue function of weighted Lagrange interpolation-I. (Freud-type weights)," Constructive Approximation, vol. 15, no. 3, pp. 355-367, 1999.

[33] J. A. C. Weideman, "Spectral methods based on nonclassical orthogonal polynomials," in Applications and Computation of Orthogonal Polynomials, vol. 131 of International Series of Numerical Mathematics, pp. 239-251, Birkhäuser, Basel, Switzerland, 1999.

[34] H. Chen and B. D. Shizgal, "A spectral solution of the Sturm-Liouville equation: comparison of classical and nonclassical basis sets," Journal of Computational and Applied Mathematics, vol. 136, no. 1-2, pp. 17-35, 2001.

[35] A. Alipanah, M. Razzaghi, and M. Dehghan, "Nonclassical pseudospectral method for the solution of brachistochrone problem," Chaos, Solitons and Fractals, vol. 34, no. 5, pp. 1622-1628, 2007.

[36] G. Criscuolo, B. Della Vecchia, D. S. Lubinsky, and G. Mastroianni, "Functions of the second kind for Freud weights and series expansions of Hilbert transforms," Journal of Mathematical Analysis and Applications, vol. 189, no. 1, pp. 256-296, 1995. 


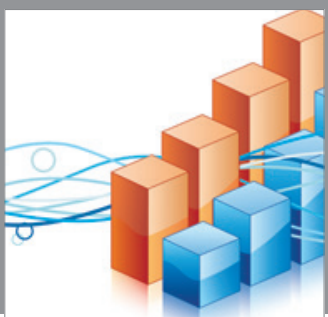

Advances in

Operations Research

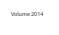

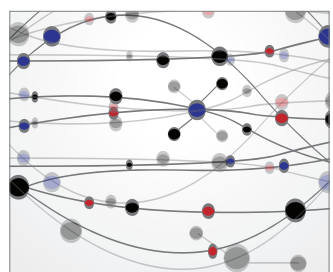

\section{The Scientific} World Journal
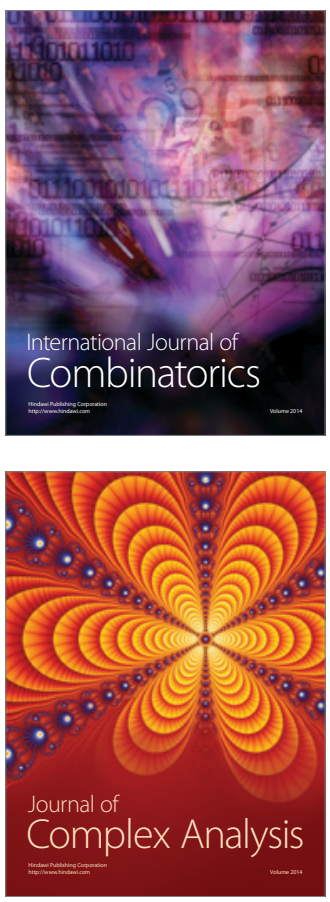

International Journal of

Mathematics and

Mathematical

Sciences
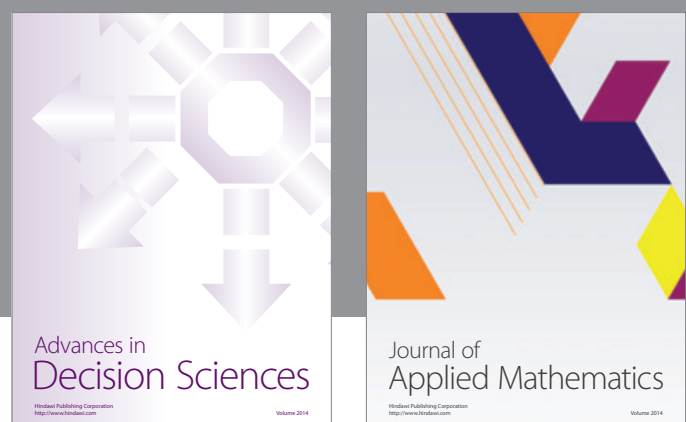

Journal of

Applied Mathematics
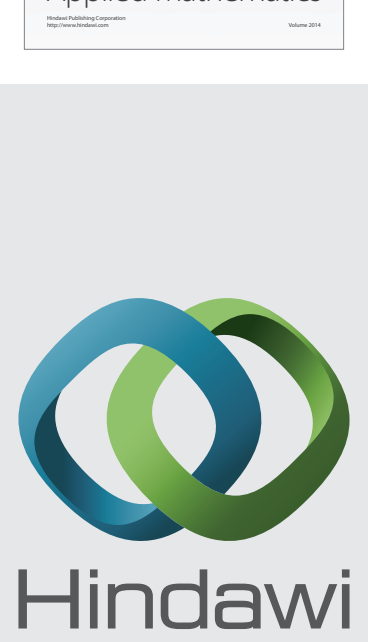

Submit your manuscripts at http://www.hindawi.com
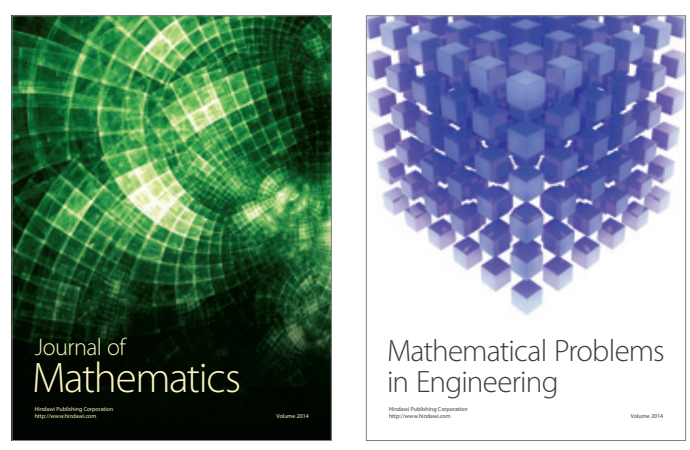

Mathematical Problems in Engineering
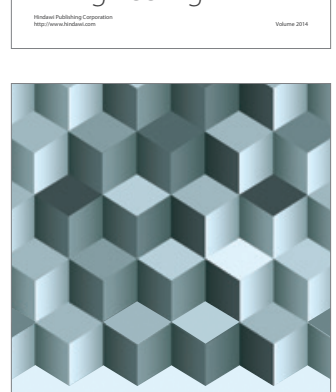

Journal of

Function Spaces
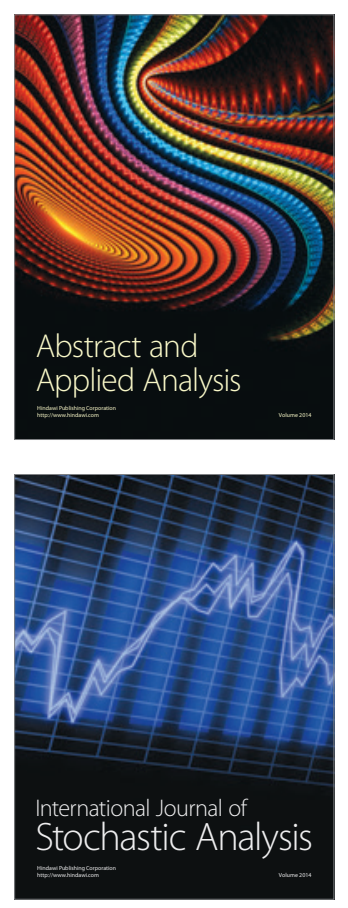

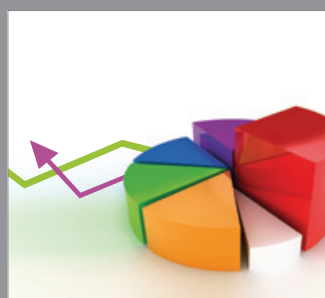

ournal of

Probability and Statistics

Promensencen
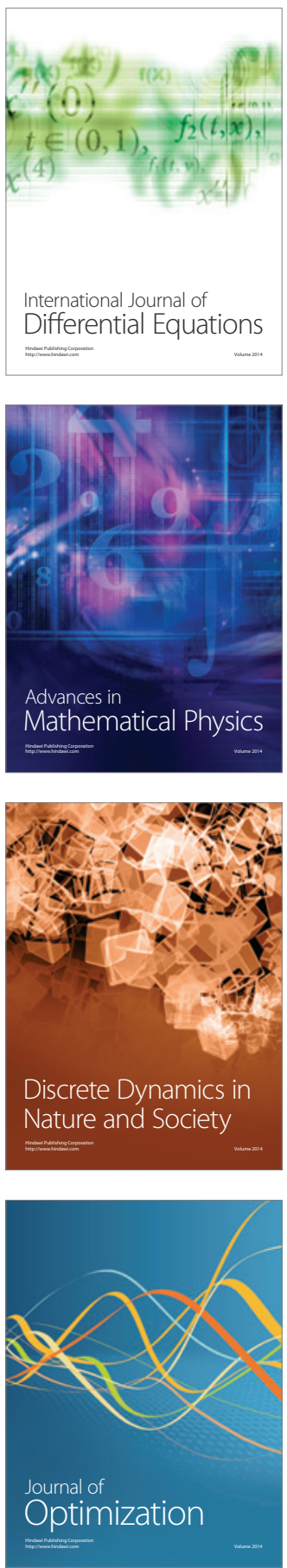